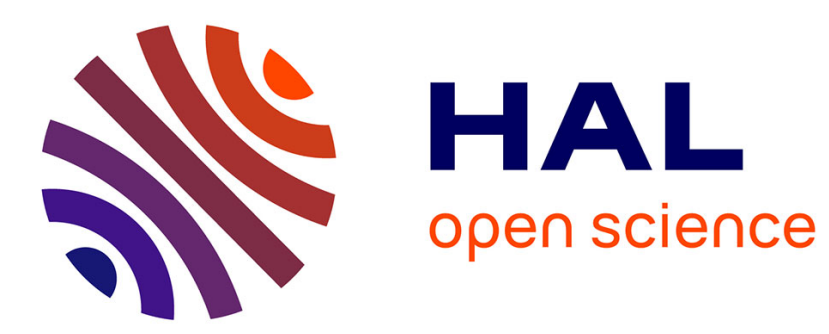

\title{
Distributed Control of Nonlinear Diffusion Systems by Input-Output Linearization
}

\author{
Ahmed Maidi, Jean-Pierre Corriou
}

\section{To cite this version:}

Ahmed Maidi, Jean-Pierre Corriou. Distributed Control of Nonlinear Diffusion Systems by Input-Output Linearization. International Journal of Robust and Nonlinear Control, 2012, 10.1002/rnc.2892 . hal-02383525

\section{HAL Id: hal-02383525 \\ https://hal.science/hal-02383525}

Submitted on 2 Dec 2019

HAL is a multi-disciplinary open access archive for the deposit and dissemination of scientific research documents, whether they are published or not. The documents may come from teaching and research institutions in France or abroad, or from public or private research centers.
L'archive ouverte pluridisciplinaire HAL, est destinée au dépôt et à la diffusion de documents scientifiques de niveau recherche, publiés ou non, émanant des établissements d'enseignement et de recherche français ou étrangers, des laboratoires publics ou privés. 


\section{Distributed Control of Nonlinear}

\section{Diffusion Systems by Input-Output}

\section{Linearization}

Ahmed MAIDI $^{a}$, and Jean-Pierre CORRIOU ${ }^{b *}$

${ }^{a}$ Laboratoire de Conception et Conduite des Systèmes de Production, Université Mouloud MAMMERI, 15000 Tizi-Ouzou, Algérie.

${ }^{b}$ Laboratoire Réactions et Génie des Procédés,

UMR 7274-CNRS, Lorraine Université, ENSIC

1, rue Grandville, BP 20451, 54001 Nancy Cedex, France.

December 2, 2019

${ }^{*}$ Corresponding author: jean-pierre.corriou@univ-lorraine.fr 
This paper addresses the distributed control by input-output linearization of a non linear diffusion equation, which describes a particular but important class of distributed parameter systems. Both manipulated and controlled variables are assumed to be distributed in space. The control law is designed using the concept of characteristic index from geometric control by using directly the PDE model without any approximation or reduction. The main idea consists in the control design in assuming an equivalent linear diffusion equation obtained by use of the Cole-Hopf transformation. This framework helps to demonstrate the closed-loop stability using some concepts from the powerful semi-group theory. The performance of the proposed controller is successfully tested, through simulation, by considering a nonlinear heat conduction problem concerning the control of the temperature of a steel plate modeled by a non linear heat equation with Dirichlet boundary conditions.

Keywords:distributed parameter system, diffusion system, Cole-Hopf transformation, geometric control, semi-group theory, exponential stability 


\section{Introduction}

The dynamic behavior of most distributed parameter systems (DPS) is described by partial nonlinear differential equations (PDE). Control of DPS occupies an important place in control theory and constitutes an active research area $[1,2]$. Compared to the control theory for linear DPS, which has been attained a certain level of maturity thanks to semi-group theory [3, 4], the control theory of nonlinear DPS remains very challenging and many interesting questions are open concerning control, stabilization and optimization [1]. The book by Chen et al. [5] gives recent progress in control theory of nonlinear systems and includes many results, applications and literature citations.

Although, in recent years, an extensive effort has been made to investigate the control of nonlinear DPS, however the available results, often developed under some restrictive assumptions, are difficult to generalize and are valid for specified particular classes, which limits their use [1, 2].

Control design methodologies of a non linear distributed system can be split into two approaches $[6,7]$. The first one called early lumping represents the conventional approach. It consists in performing a reduction of the PDE to derive a set of ordinary differential equations (ODE) that constitute an 
approximation, or a reduction, of the original PDE model $[8,9]$, and the controller design is performed in the framework of the classical control theory of lumped parameter systems (LPS) using potentially powerful techniques. It must be noted that through early lumping, the fundamental control theoretical properties (controllability, observability and stability) are lost [6, 7]. This approach, generally, leads to high dimension controllers which are difficult to implement [6]. The second approach, termed as late lumping, uses the PDE model for the controller design without approximation. The approximation is performed only for implementation purposes of the controller. Late lumping allows the control designer to avoid losing the distributed nature of the PDE system and to take full advantage of their natural properties. However, direct handling of PDEs is difficult and the hardest task is related to the proof of the control theoretical properties, which needs sophisticated mathematical tools. In recent years, several control methods that directly take into account the distributed nature of the systems have been developed especially for the linear case $[3,4,10]$ and quasi-linear system [6].

For nonlinear distributed parameter systems, the examination of the literature dedicated to the control problem of this kind of systems reveals that most contributions come from the early lumping approach and address the 
controller synthesis problem on the basis of low dimensional ODE approximations of the PDE system (reduced model) using different reduction approaches [11] to achieve an accurate reduced model that characterizes the dominant dynamic behavior $[9,8,12,13,14,15]$. On the other hand, few applications of the late lumping approach have been reported in the literature [16] and most of them are developed in the framework of geometric control $[17,18,19,20,21,22,23]$, and reveal that geometric control is an interesting and suitable approach for designing controllers for distributed parameter following the late lumping approach. The most important advantages of geometric control is that the control law can be designed directly using the PDE model, which leads to distributed control that increases the performances [6]. In addition, the geometric control allows to take advantage of the full potential of an existing control theory for lumped linear systems that offers full powerful controller design techniques. Nevertheless, the difficulty for the geometric control of nonlinear systems is that the closed-loop stability is difficult to prove and needs some sophisticated mathematical tools from functional analysis. Geometric control has been applied with success for linear and quasi-linear systems under some assumptions related to the system characteristics $[17,18,19,20,21,22,23]$. An attempt to extend the geometric 
approach to nonlinear systems, with boundary actuation and punctual controlled variable, is proposed by Maidi and Corriou [24]. As the control and observation operators assumed are unbounded, this approach suffers from the lack of efficient tools in proving closed-loop stability.

The investigations of the present work are intended as a contribution to the geometric control of nonlinear diffusive systems, which is one of the most important classes encountered in a wide variety of practical applications [14]. Thus, a design approach of the control law that enforces the desired performance and stability is developed. The main idea consists in deriving a linear equivalent model of the nonlinear diffusion system by use of the Cole-Hopf transformation. This linear equivalent model will be used then for control design and in particular for the proof of closed-loop stability using some concepts from semi-group theory. The developed control method is illustrated through a heat conduction problem modeled by a nonlinear diffusion equation.

The present paper is structured as follows. In Section 2, the addressed control problem for a nonlinear diffusion system is presented. In Section 3, the equivalent linear model is derived by means of Cole-Hopf transformation. Section 4 contains the main results associated with the control design 
approach proposed and the stability analysis based on the semi-group theory. In Section 5, the performance of the designed controller following the proposed approach is evaluated through simulation studies performed in an illustrative example concerning the heating problem of a steel plate with a nonconstant thermal conductivity. Finally, concluding remarks are provided in Section 6.

\section{Control problem formulation}

The nonlinear class of DPS, considered here, are described by the onedimensional nonlinear diffusion equation with interior control distributed on the space domain. The corresponding PDE model is

$$
\left.\frac{\partial x(z, t)}{\partial t}=\frac{1}{\rho c_{p}}\left[\frac{\partial}{\partial z}\left(k(x(z, t)) \frac{\partial x(z, t)}{\partial z}\right)+\vartheta(z, t)\right] \quad \text { in } \Omega \times\right] 0, t[
$$

accompanied by Dirichlet boundary conditions

$$
\left.x(0, t)=x(l, t)=x^{*} \quad \text { in } \partial \Omega \times\right] 0, t[
$$


and the initial condition

$$
x(z, 0)=\phi(z) \quad \text { in } \Omega
$$

where $x(z, t)$ denotes the state, $z \in \Omega=[0, l] \subset \Re$ is the spatial domain, $\partial \Omega$ are the spatial domain boundaries, $t \in[0, \infty[$ is the time variable, $k(x(z, t))>0$ is the conductivity, and $\rho c_{p}$ the capacity of fluid or solid (in the case of solids, $c$ remplaces $c_{p}$ ). Without loss of generality and to simplify the presentation, the terminology from heat conduction transfer will be used. Thus, $k(x(z, t))$ and $\rho c_{p}$ will denote the thermal conductivity and the heat capacity, respectively.

The main space is defined as $L^{2}(0, l)$, which is the space of squareintegrable functions on $\Omega=[0, l]$, endowed with the usual norm $\|\cdot\|_{L^{2}(0, l)}$ and the inner product $\langle,\rangle_{L^{2}(0, l)}$ defined as

$$
\begin{aligned}
\langle f, g\rangle_{L^{2}(0, l)} & =\int_{0}^{l} f(z) g(z) d z ; \quad \forall f, g \in L^{2}(0, l) \\
\|f\|_{L^{2}(0, l)} & =\langle f, f\rangle_{L^{2}(0, l)}^{\frac{1}{2}}
\end{aligned}
$$

The distributed manipulated variable $\vartheta(z, t) \in L^{2}\left(\left[0, \infty\left[; L^{2}(0,1)\right)\right.\right.$ is 
given as follows :

$$
\vartheta(z, t)=b(z) u(t)
$$

where $b(z)$ is a known smooth function of $z$ assumed to be square integrable on the interval $[0, l]$ that is $b(z) \in L^{2}(0, l)$.

The output variable $y(t)$ to be controlled by manipulating the uniformly distributed control $u(t) \in L^{2}([0, \infty[; \Re)$ is

$$
y(t)=\mathcal{C} x(z, t)=\int_{0}^{l} c(z) x(z, t) d z ; \quad \mathcal{C} \in \mathcal{L}\left(L^{2}(0, l) ; \Re\right)
$$

where $\mathcal{C}$ is a bounded linear operator, $\mathcal{L}$ is the space of all linear bounded operators from $L^{2}(0, l)$ into $\Re$, and $c(z)$ is a known smooth function of $z$. In practice, the selection of $b(z)$ and $c(z)$ is typically consistent, therefore the following assumptions will be made

$$
c(z) \in H^{2}(0, l)
$$

and

$$
c(0)=c(l)=0
$$

where $H^{2}(0, l)$ denotes a Hilbert space defined as the Sobolev space of order 
$2[25,26]$, i.e.

$$
H^{2}(0, l)=\left\{c(z) \in L^{2}(0, l): \frac{d^{k} c(z)}{d z^{k}} \in L^{2}(0, l) ; k=1,2\right\}
$$

The two functions $b(z)$ and $c(z)$ are assumed not orthogonal, thus

$$
\langle b(z), c(z)\rangle_{L^{2}(0, l)}=\int_{0}^{l} b(z) c(z) d z \neq 0
$$

Remark 1 The control problem is formulated with Dirichlet boundary conditions but the following development still holds for other types of boundary conditions.

Remark 2 In the control problem formulated, a single spatial interval $[0, l]$ is considered but it can be formulated as the problem of controlling the output $y(t)$ at a finite number of spatial intervals as suggested by Christofides [17]. For this case, the control law proposed here remains valid.

\section{Cole-Hopf transformation}

The Cole-Hopf transformation is widely used for solving nonlinear diffusion equation $[27,28,29]$ but for control problems, it has not yet been exploited. 
This transformation consists in converting the nonlinear diffusion equation into a linear one if the thermal diffusivity defined by the following ratio

$$
\alpha=\frac{k(x(z, t))}{\rho c_{p}}
$$

is approximately constant. This hypothesis is accepted since in many cases the variation of $\alpha$ with $x(z, t)$ is much less important than that of $k(x(z, t))$, so that this approximation is reasonable [30], therefore $\alpha \approx$ constant.

To linearize the nonlinear diffusion equation (1) using the Cole-Hopf technique, one seeks a transformation of the form [27]

$$
x(z, t)=h(w(z, t))
$$

where $h(x(z, t))$ is a continuous bijective function (one-to-one function or mapping).

By using the transform (13), the evaluation of the derivatives of the non- 
linear diffusion equation (1) gives

$$
\begin{aligned}
\frac{\partial x(z, t)}{\partial t} & =\frac{\partial h(w(z, t))}{\partial t} \\
& =\frac{\partial h(w(z, t))}{\partial w(z, t)} \frac{\partial w(z, t)}{\partial t}
\end{aligned}
$$

and

$$
\begin{aligned}
\frac{\partial}{\partial z}\left(k(x(z, t)) \frac{\partial x(z, t)}{\partial z}\right)= & \frac{\partial}{\partial z}\left(k(h(w(z, t))) \frac{\partial h(w(z, t))}{\partial z}\right) \\
& =\left[k(h(w(z, t))) \frac{d^{2} h(w(z, t))}{d w^{2}(z, t)}+\frac{d k(h(w(z, t)))}{d h(w(z, t))}\left(\frac{d h(w(z, t))}{d w(z, t)}\right)^{2}\right] \\
& \left(\frac{\partial w(z, t)}{\partial z}\right)^{2}+k(h(w(z, t))) \frac{d h(w(z, t))}{d w(z, t)} \frac{\partial^{2} w(z, t)}{\partial z^{2}}
\end{aligned}
$$

To make the right hand side of (15) linear, the term between square brackets is set equal to zero,

$$
k(h(w(z, t))) \frac{d^{2} h(w(z, t))}{d w^{2}(z, t)}+\frac{d k(h(w(z, t)))}{d h(w(z, t))}\left(\frac{d h(w(z, t))}{d w(z, t)}\right)^{2}=0
$$

this differential equation can be expressed in the following integrable form

$$
\frac{d}{d w(z, t)}\left[k(h(w(z, t))) \frac{d h(w(z, t))}{d w(z, t)}\right]=0
$$


Integrating (17) gives

$$
k(h(w(z, t))) \frac{d h(w(z, t))}{d w(z, t)}=c_{1}
$$

hence

$$
\begin{aligned}
\int k(h(w(z, t))) d h(w(z, t)) & =\int c_{1} d w(z, t) \\
& =c_{1} w(z, t)+c_{2}
\end{aligned}
$$

or equivalently

$$
w(z, t)=\frac{1}{c_{1}} \int k(x(z, t)) d x(z, t)-\frac{c_{2}}{c_{1}}
$$

which can be written under the following form

$$
w(z, t)=h^{-1}(x(z, t))=\frac{1}{c_{1}} \int k(x(z, t)) d x(z, t)-\frac{c_{2}}{c_{1}}
$$

where $h^{-1}($.$) is the inverse function of h($.$) , which is continuous.$

In summary, by using the transformation (13) with $h$ satisfying (16), the 
nonlinear diffusion equation (1) will be converted to the following linear one

$$
\begin{aligned}
\frac{\partial h(w(z, t))}{\partial w(z, t)} \frac{\partial w(z, t)}{\partial t} & =\frac{k(x(z, t))}{\rho c_{p}} \frac{\partial h(w(z, t))}{\partial w(z, t)} \frac{\partial^{2} w(z, t)}{\partial z^{2}}+\frac{1}{\rho c_{p}} \vartheta(z, t) \\
\frac{\partial w(z, t)}{\partial t} & =\frac{k(x(z, t))}{\rho c_{p}} \frac{\partial^{2} w(z, t)}{\partial z^{2}}+\frac{1}{\rho c_{p} \frac{\partial h(w(z, t))}{\partial w(z, t)}} \vartheta(z, t)
\end{aligned}
$$

Considering the expressions of diffusivity $\alpha$ and the integration constant $c_{1}$ given by (12) and (18), respectively, equation (22) takes the following form

$$
\frac{\partial w(z, t)}{\partial t}=\alpha \frac{\partial^{2} w(z, t)}{\partial z^{2}}+\frac{\alpha}{c_{1}} \vartheta(z, t)
$$

and according to (2), the transformation (20), with appropriate choice of the integration constants $c_{1}$ and $c_{2}$, yields the inhomogeneous boundary conditions

$$
w(0, t)=w(l, t)=0
$$

with the initial condition

$$
w(z, 0)=0
$$


and the controlled output is

$$
y(t)=\mathcal{C} h(w(z, t))
$$

Remark 3 The Kirchhoff transformation is a particular case of the ColeHopf transformation [27]. Compared to the Kirchhoff transformation that uses a definite integral, the Cole-Hopf uses an indefinite integral, which allows the adjustment of the transform by choosing adequate integration constants $c_{1}$ and $c_{2}$. Consequently, desirable and simple practical transformation that ensures homogeneous boundary conditions can be obtained using the ColeHopf technique. The Kirchhoff transformation is obtained by choosing $c_{1}=k_{0}$ $\left(k_{0}=k(x(z, 0))=k\left(x_{0}\right)\right)$ and $c_{2}=-\frac{1}{k_{0}}\left[\int k(x(z, t)) d x(z, t)\right]_{x(z, t)=x 0}[$ [27] . Thus,

$$
\begin{aligned}
w(z, t) & =\frac{1}{k_{0}}\left[\int k(x(z, t)) d x(z, t)\right]_{x(z, t)=x(z, t)}-\frac{1}{k_{0}}\left[\int k(x(z, t)) d x(z, t)\right]_{x(z, t)=x_{0}} \\
& =\frac{1}{k_{0}} \int_{x 0}^{x(z, t)} k(x(z, t)) d x(z, t)
\end{aligned}
$$

Remark 4 The mapping $h(x(z, t))$ is a continuous bijective function (oneto-one function), thus its inverse function $h^{-1}(x(z, t))$ exists and is also con- 
tinuous.

Remark 5 For control problem design, the determination of transformation $h(x(z, t))$ is not necessary, but for proving the closed-loop stability, its continuity property will be exploited.

\section{Distributed feedback design}

The design of the control law $u(t)$ of the control problem formulated is performed in the framework of geometric control using the concept of characteristic index $[6,17]$, which is a generalization of the concept of relative degree $[31,32]$ used in lumped parameter systems (ODE systems) to PDE systems. The characteristic index is the smallest order of the time derivative of a given controlled variable which explicitly depends on the manipulated variable.

Considering the distributed control (6), the linear diffusion equation (23) can be written under the following form

$$
\frac{\partial w(z, t)}{\partial t}=\mathcal{A} w(z, t)+\mathcal{B} u(t)
$$


where $\mathcal{A}$ and $\mathcal{B}$ are the following operators

$$
\mathcal{A}=\alpha \frac{\partial^{2}}{\partial z^{2}} \quad ; \quad \mathcal{B}=\frac{\alpha}{c_{1}} b(z)
$$

\subsection{Control law design}

For the synthesis of the geometric control law, the linear diffusion equation

(28) will be considered. Hence, the first derivative of the controlled output (26) is

$$
\begin{aligned}
\frac{d y(t)}{d t} & =\mathcal{C}\left\{\frac{\partial h(w(z, t))}{\partial t}\right\} \\
& =\mathcal{C}\left\{\frac{d h(w(z, t))}{d w(z, t)} \frac{\partial w(z, t)}{\partial t}\right\} \\
& =\frac{c_{1}}{\alpha \rho c_{p}} \mathcal{C}\left\{\frac{\partial w(z, t)}{\partial t}\right\} \\
& =\frac{c_{1}}{\alpha \rho c_{p}} \mathcal{C}[\mathcal{A} w(z, t)+\mathcal{B} u(t)] \\
& =\frac{c_{1}}{\alpha \rho c_{p}} \mathcal{C} \mathcal{A} w(z, t)+\frac{c_{1}}{\alpha \rho c_{p}} \mathcal{C B} u(t)
\end{aligned}
$$

The manipulated input $u(t)$ appears linearly in the first time derivative of the output. The development of the second term of the right-hand side of 
equation (31) leads to

$$
\begin{aligned}
\frac{c_{1}}{\alpha \rho c_{p}} \mathcal{C B} u(t) & =\frac{c_{1}}{\alpha \rho c_{p}} \int_{0}^{l} c(z) \frac{\alpha}{c_{1}} b(z) u(t) d z \\
& =\frac{1}{\rho c_{p}}\left\{\int_{0}^{l} c(z) b(z) d z\right\} u(t) \\
& =\frac{1}{\rho c_{p}}\langle b(z), c(z)\rangle_{L^{2}(0, l)} u(t)
\end{aligned}
$$

as $b(z)$ and $c(z)$ are not orthogonal, hence

$$
\frac{1}{\rho c_{p}}\langle b(z), c(z)\rangle_{L^{2}(0, l)} \neq 0
$$

consequently, the characteristic index is $\sigma=1$, which suggests requesting the following input-output response for the closed-loop system (between the controlled output $y(t)$ and the reference input $v(t))$

$$
\tau \frac{d y(t)}{d t}+y(t)=v(t)
$$

where $\tau$ is the desired time constant of the closed-loop system.

Thus, substituting (31) into equation (35), the distributed state-feedback 
control law can be easily deduced as follows

$$
u(t)=\frac{\rho c_{p}}{\tau \int_{0}^{l} b(z) c(z) d z}\left[v(t)-y(t)-\frac{\tau c_{1}}{\alpha \rho c_{p}} \mathcal{C} \mathcal{A} w(z, t)\right]
$$

Let us express the control law $u(t)$ according to the state $x(z, t)$. Remember that the expression (16) is made equal to zero by the transformation (13), thus considering the relations (15) and (18), the term $\mathcal{A} w(z, t)$ of the control law (36) can be written as

$$
\begin{aligned}
\mathcal{A} w(z, t) & =\alpha \frac{\partial^{2} w(z, t)}{\partial z^{2}} \\
& =\frac{\alpha}{c_{1}} \frac{\partial}{\partial z}\left(k(x(z, t)) \frac{\partial x(z, t)}{\partial z}\right)
\end{aligned}
$$

and the control law (36) takes the following form

$$
\begin{aligned}
u(t) & =\frac{\rho c_{p}}{\tau \int_{0}^{l} b(z) c(z) d z}\left[v(t)-y(t)-\frac{\tau}{\rho c_{p}} \mathcal{C}\left\{\frac{\partial}{\partial z}\left(k(x(z, t)) \frac{\partial x(z, t)}{\partial z}\right)\right\}\right] \\
& =\frac{\rho c_{p}}{\tau \int_{0}^{l} b(z) c(z) d z}\left[v(t)-y(t)-\frac{\tau}{\rho c_{p}} \int_{0}^{l} c(z) \frac{\partial}{\partial z}\left(k(x(z, t)) \frac{\partial x(z, t)}{\partial z}\right) d z\right]
\end{aligned}
$$

Remark 6 The control law (38) can be directly derived by considering the 
nonlinear diffusion equation (1) in evaluating the first derivative of $y(t)$ given by (7). In this case, it is difficult to show the closed-loop stability since both system (1) and control law (38) are nonlinear. Thus, the purpose of designing of the control law based on the linear diffusion equation (23) is to prove the closed-loop stability using some concepts from semi-group theory as it is shown in the next subsection.

Remark 7 The control law (38) is infinite-dimensional, thus for simulation purpose or for on-line implementation, a finite-dimensional approximation of the control law (38) has to be derived using discretization methods, such as finite differences. According to Balas [33], to ensure the convergence of the closed-loop system resulting from the PDE model plus a finite-dimensional approximation of the infinite-dimensional control law to the closed-loop system resulting from the PDE model plus the infinite dimensional control law, the number of discretization points must be increased.

Remark 8 The control law (38) allows the application of the linear control theory to the resulting linear reference input $v(t)$-controlled output $y(t)$ system (35) using powerful design approaches developed for linear lumped parameter systems. Thus, to handle uncertainty and unmodeled dynamics, the reference input $v(t)$ can be defined by means of a robust controller [31, 32, 34, 35], that 
is

$$
v(t)=\int_{0}^{t} G(t-\xi)\left[y^{d}(t)-y(t)\right] d \xi
$$

where $y^{d}(t)$ is the desired set point of the controlled output $y(t)$ and the function $G($.$) , for instance, can be chosen as the inverse of an appropriate trans-$ fer function.

This strategy has been applied with success for a counter-current heat exchanger [20], a parallel-flow heat exchanger [22] and for a wave equation [23].

Remark 9 The control law design approach proposed is developed for controlling the output (7) defined as the spatial weighted average. In this case, the control and observation operators $\mathcal{B}$ and $\mathcal{C}$, respectively are bounded. The proposed control law is still applicable in the case of a punctual output $y_{p}(t)$ by adopting the control strategy proposed by [22, 24] where an external robust controller is introduced to provide the desired reference for the internal controller (see remark 8), that is $y^{d}(t)$, by taking the error between the controlled punctual output $y_{p}(t)$ and its set point $y_{p}^{d}(t)$, i.e.

$$
y^{d}(t)=\int_{0}^{t} G_{p}(t-\xi)\left[y_{p}^{d}(t)-y_{p}(t)\right] d \xi
$$


For more details about this strategy, the reader is referred to Maidi et al. [22] and Maidi and Corriou [24].

\subsection{Closed-loop stability}

The equivalent closed-loop system is given by

$$
\begin{aligned}
\frac{\partial w(z, t)}{\partial t} & =\mathcal{A} w(z, t)+\mathcal{B}\left\{\frac{\rho c_{p}}{\tau \int_{0}^{l} b(z) c(z) d z}\left[v(t)-y(t)-\frac{\tau c_{1}}{\alpha \rho c_{p}} \mathcal{C} \mathcal{A} w(z, t)\right]\right\} \\
& =(\mathcal{A}+\mathcal{F}) w(z, t)+\frac{\rho c_{p}}{\tau \int_{0}^{l} b(z) c(z) d z} \mathcal{B}\{v(t)-y(t)\} \\
y(t) & =\mathcal{C} h(w(z, t))
\end{aligned}
$$

where

$$
\mathcal{F}=\beta b(z) \mathcal{C A}, \quad \beta=\left[-\int_{0}^{l} b(z) c(z) d z\right]^{-1}
$$

By constraining the reference input $v(t)$ and the controlled output $y(t)$ to zero $(y(t)=\mathcal{C} h(w(z, t))=0, v(t)=0)$, the zero dynamics associated with the open-loop system results

$$
\begin{aligned}
\frac{\partial w(z, t)}{\partial t} & =(\mathcal{A}+\mathcal{F}) w(z, t) \\
y(t) & \equiv 0
\end{aligned}
$$


with boundary conditions (24).

If the operator $\mathcal{A}+\mathcal{F}$ generates a stable semi-group, it implies that the zero dynamics is exponentially stable $[6,17]$. Thus, according to theorem 1 given in appendix 7.1, the operator $\mathcal{A}+\mathcal{F}$ generates a semi-group if the operator $\mathcal{A}$ is a generator of a semi-group and the operator $\mathcal{F}$ is bounded on $L^{2}(0, l)$.

The operator $\mathcal{A}$ with the boundary conditions (24) and initial condition (25) generates an exponentially stable semi-group $U(t)[3,36]$, that is,

$$
\|U(t)\|_{L^{2}(0, l)} \leq M e^{-\omega t}
$$

with stability constants $M=1$ and $\omega=\alpha \pi^{2}>0$.

Let us, now, demonstrate that the operator $\mathcal{F}$ is bounded. According to appendix 7.3, the operator $\mathcal{F}$ is bounded if there exists a constant $C$ such that

$$
\begin{gathered}
\|\mathcal{F} w(z, t)\|_{L^{2}(0, l)} \leq C\|w(z, t)\|_{L^{2}(0, l)} \\
\|\mathcal{F} w(z, t)\|_{L^{2}(0, l)}=\|\beta b(z) \mathcal{C} \mathcal{A} w(z, t)\|_{L^{2}(0, l)} \\
=\left\|\beta \alpha b(z) \int_{0}^{l} c(z) \frac{\partial^{2} w(z, t)}{\partial z^{2}} d z\right\|_{L^{2}(0, l)}
\end{gathered}
$$


Now, by integrating by parts and by considering boundary conditions (9) and (24), the calculus gives

$$
\begin{aligned}
\|\mathcal{F} w(z, t)\|_{L^{2}(0, l)}^{2} & =\left\|\beta \alpha b(z) \int_{0}^{l} \ddot{c}(z) w(z, t) d z\right\|_{L^{2}(0, l)}^{2} \\
& =\int_{0}^{l}\left[\beta \alpha b(z) \int_{0}^{l} \ddot{c}(z) w(z, t) d z\right]^{2} d z \\
& =\beta^{2} \alpha^{2} \int_{0}^{l}\left[b(z)\left|\int_{0}^{l} \ddot{c}(z) w(z, t) d z\right|\right]^{2} d z
\end{aligned}
$$

and the Cauchy-Schwarz inequality [26] yields

$$
\left|\int_{0}^{l} \ddot{c}(z) w(z, t) d z\right| \leq\|\ddot{c}(z)\|_{L^{2}(0, l)}\|w(z, t)\|_{L^{2}(0, l)}
$$

hence

$$
\begin{aligned}
\|\mathcal{F} w(z, t)\|_{L^{2}(0, l)}^{2} & \leq \beta^{2} \alpha^{2} \int_{0}^{l} b^{2}(z)\left[\|\ddot{c}(z)\|_{L^{2}(0, l)}\|w(z, t)\|_{L^{2}(0, l)}\right]^{2} d z \\
& =\beta^{2} \alpha^{2} \int_{0}^{l} b^{2}(z)\|\ddot{c}(z)\|_{L^{2}(0, l)}^{2}\|w(z, t)\|_{L^{2}(0, l)}^{2} d z \\
& =\beta^{2} \alpha^{2} \int_{0}^{l} b^{2}(z)\left[\int_{0}^{l}[\ddot{c}(z)]^{2} d z\right]\left[\int_{0}^{l} w^{2}(z, t) d z\right] d z \\
& =\beta^{2} \alpha^{2}\left[\int_{0}^{l}[\ddot{c}(z)]^{2} d z\right]\left[\int_{0}^{l} w^{2}(z, t) d z\right] \int_{0}^{l} b^{2}(z) d z
\end{aligned}
$$


thus

$$
\begin{aligned}
\|\mathcal{F} w(z, t)\|_{L^{2}(0, l)}^{2} & \leq \beta^{2} \alpha^{2} \gamma \int_{0}^{l} w^{2}(z, t) d z \\
& \leq \beta^{2} \alpha^{2} \gamma\|w(z, t)\|_{L^{2}(0, l)}^{2}
\end{aligned}
$$

with $\gamma=\left[\int_{0}^{l}[\ddot{c}(z)]^{2} d z\right]\left[\int_{0}^{l} b^{2}(z) d z\right]$.

From (51), it follows that

$$
\|\mathcal{F} w(z, t)\|_{L^{2}(0, l)} \leq|\beta| \alpha \gamma^{1 / 2}\|w(z, t)\|_{L^{2}(0, l)}
$$

Recall that $b(z) \in L^{2}(0, l)$ and $c(z) \in H^{2}(0, l)$, hence the constant $C=$ $|\beta| \alpha \gamma^{1 / 2}$ exists, which means that $\mathcal{F}$ is bounded and $\|\mathcal{F}\|_{L^{2}(0, l)}=|\beta| \alpha \gamma^{1 / 2}$. Now, according to the theorem 2 given in appendix 7.2 [37, Theorem 1.1, page 76], the operator $\mathcal{A}+\mathcal{F}$ generates a semi-group $V(t)$ such that

$$
\begin{aligned}
\|V(t)\|_{L^{2}(0, l)} & \leq M e^{\left(-\omega+M\|\mathcal{F}\|_{L^{2}(0, l)}\right) t} \\
& \leq M e^{\left(-\omega+M|\beta| \alpha \gamma^{1 / 2}\right) t} \\
& \leq e^{\left(-\alpha \pi^{2}+|\beta| \alpha \gamma^{1 / 2}\right) t} \\
& \leq e^{-\left(\alpha \pi^{2}-|\beta| \alpha \gamma^{1 / 2}\right) t}
\end{aligned}
$$


thus the semigroup $V(t)$ is exponentially stable if the following stability constant

$$
\omega_{w}=\alpha \pi^{2}-|\beta| \alpha \gamma^{1 / 2}
$$

is positive, which yields

$$
|\beta| \gamma^{1 / 2}<\pi^{2}
$$

This implies that the exponential stability of $V(t)$ is related to the choice of the functions $b(z)$ and $c(z)$ since both $\beta$ and $\gamma$ depend on these functions. Consequently, in addition to the controllability condition ensured by (11), the functions $b(z)$ and $c(z)$ should be chosen so that the stability condition (55) is verified. In this case, the zero dynamics is exponentially stable and the operator $\mathcal{A}+\mathcal{F}$ generates an exponentially stable semi-group $V(t)$. In the following development, it is assumed that $V(t)$ is stable.

Now, the closed-loop system (41-42) can be written in the form of the following interconnected $y$-subsystem and $w$-subsystem

$$
\begin{aligned}
\dot{y}(t) & =-\frac{1}{\tau} y(t)+\frac{1}{\tau} v(t) \\
\frac{\partial w(z, t)}{\partial t} & =(\mathcal{A}+\mathcal{F}) w(z, t)+\frac{\alpha \rho c_{p}}{c_{1} \tau \int_{0}^{l} b(z) c(z) d z} b(z) W(t)
\end{aligned}
$$


where $W(t)=v(t)-y(t)$.

To verify the closed-loop stability, it is equivalent to verify that the cascade interconnection is stable. Thus, it is sufficient to verify the closed-loop stability of each subsystem, which is demonstrated in the following.

According to (35), as the time constant $\tau>0$, thus the $y$-subsystem (56) of the interconnection is exponentially stable, consequently

$$
|W(t)| \leq K_{W}|W(0)| e^{-\omega_{W} t}, \quad K_{W} \geq 1 \text { and } \omega_{W}>0
$$

with $W(0)=v(0)-y(0)$.

Now, as the semi-group $V(t)$, generated by the operator $\mathcal{A}+\mathcal{F}$, is exponentially stable, consequently, the state $w(z, t)$ of the closed-loop system (41-42) verifies [3]

$$
\begin{aligned}
\|w(z, t)\|_{L^{2}(0, l)} & \leq\|w(z, 0)\|_{L^{2}(0, l)} e^{-\omega_{w} t}+\int_{0}^{t} e^{-\omega_{w}(t-\xi)} \frac{\alpha \rho c_{p}\|b(z)\|_{L^{2}(0, l)}}{c_{1} \tau \int_{0}^{l} b(z) c(z) d z}|W(\xi)| d \xi \\
& \leq\|w(z, 0)\|_{L^{2}(0, l)} e^{-\omega_{w} t}+\frac{\alpha \rho c_{p}\|b(z)\|_{L^{2}(0, l)}}{c_{1} \tau \int_{0}^{l} b(z) c(z) d z} \int_{0}^{t} e^{-\omega_{w}(t-\xi)}|W(\xi)| d \xi
\end{aligned}
$$

where $\omega_{w}=\alpha \pi^{2}-|\beta| \alpha \gamma^{1 / 2}$. 
Substituting $|W(\xi)|$ by its expression (58) in (59) gives

$$
\begin{aligned}
\|w(z, t)\|_{L^{2}(0, l)} & \leq\|w(z, 0)\|_{L^{2}(0, l)} e^{-\omega_{w} t}+\frac{\alpha \rho c_{p}\|b(z)\|_{L^{2}(0, l)}}{c_{1} \tau \int_{0}^{l} b(z) c(z) d z} \int_{0}^{t} e^{-\omega_{w}(t-\xi)} K_{W}|W(0)| e^{-\omega_{W} \xi} d \xi \\
& \leq\|w(z, 0)\|_{L^{2}(0, l)} e^{-\omega_{w} t}+\frac{\alpha \rho c_{p}\|b(z)\|_{L^{2}(0, l)} K_{W}|W(0)|}{c_{1} \tau \int_{0}^{l} b(z) c(z) d z} e^{-\omega_{w} t} \int_{0}^{t} e^{\left(\omega_{w}-\omega_{W}\right) \xi} d \xi
\end{aligned}
$$

Then, if $\omega_{w}=\omega_{W}$,

$$
\begin{aligned}
\|w(z, t)\|_{L^{2}(0, l)} & \leq\|w(z, 0)\|_{L^{2}(0, l)} e^{-\omega_{w} t}+\frac{\alpha \rho c_{p}\|b(z)\|_{L^{2}(0, l)} K_{W}|W(0)|}{c_{1} \tau \int_{0}^{l} b(z) c(z) d z} t e^{-\omega_{w} t} \\
& \leq\|w(z, 0)\|_{L^{2}(0, l)} e^{-\omega_{w} t}+\frac{\alpha \rho c_{p}\|b(z)\|_{L^{2}(0, l)} K_{W}|W(0)|}{c_{1} \tau \int_{0}^{l} b(z) c(z) d z} \frac{1}{\omega_{w}-\omega} e^{-\omega t}
\end{aligned}
$$

where $0<\omega<\omega_{w}$. Thus, the closed-loop system is exponentially stable. 
Now, if $\omega_{w}>\omega_{W}$,

$$
\begin{aligned}
\|w(z, t)\|_{L^{2}(0, l)} & \leq\|w(z, 0)\|_{L^{2}(0, l)} e^{-\omega_{w} t}+\frac{\alpha \rho c_{p}\|b(z)\|_{L^{2}(0, l)} K_{W}|W(0)|}{c_{1} \tau \int_{0}^{l} b(z) c(z) d z} e^{-\omega_{w} t} \frac{\left[e^{\left(\omega_{w}-\omega_{W}\right) t}-1\right]}{\omega_{w}-\omega_{W}} \\
& \leq\|w(z, 0)\|_{L^{2}(0, l)} e^{-\omega_{w} t}+\frac{\alpha \rho c_{p}\|b(z)\|_{L^{2}(0, l)} K_{W}|W(0)|}{c_{1} \tau \int_{0}^{l} b(z) c(z) d z} \frac{e^{-\omega_{W} t}-e^{-\omega_{w} t}}{\omega_{w}-\omega_{W}} \\
& \leq\|w(z, 0)\|_{L^{2}(0, l)} e^{-\omega_{w} t}+\frac{\alpha \rho c_{p}\|b(z)\|_{L^{2}(0, l)} K_{W}|W(0)|}{c_{1} \tau \int_{0}^{l} b(z) c(z) d z} \frac{e^{-\omega_{W} t}}{\omega_{w}-\omega_{W}}
\end{aligned}
$$

else if $\omega_{w}<\omega_{W}$

$$
\|w(z, t)\|_{L^{2}(0, l)} \leq\|w(z, 0)\|_{L^{2}(0, l)} e^{-\omega_{w} t}+\frac{\alpha \rho c_{p}\|b(z)\|_{L^{2}(0, l)} K_{W}|W(0)|}{c_{1} \tau \int_{0}^{l} b(z) c(z) d z} \frac{e^{-\omega_{w} t}}{\left|\omega_{w}-\omega_{W}\right|}
$$

in each case, the closed-loop system is exponentially stable. This implies that $\lim w(z, t)=0$ when $t \rightarrow \infty$.

Let us now consider the stability of the state $x(z, t)$ in closed loop, i.e. the nonlinear diffusion equation (1) with the control law (38). Thus, according to (13), $\lim x(z, t)$ when $t \rightarrow \infty$ is equal to $\lim h(w(z, t))$ when $t \rightarrow \infty$ or equivalently to $\lim h(w)$ when $w \rightarrow 0(w(z, t)$ is exponentially stable). Since $h(w(z, t))$ is a continuous bijective function, hence when $w \rightarrow 0, \lim h(w)$ 
exists that is, according to (13), $\lim x(z, t)$ when $t \rightarrow \infty$ also exists, consequently the state $x(z, t)$ is bounded which means that $x(z, t)$ is stable and the closed-loop system

$$
\begin{aligned}
\frac{\partial x(z, t)}{\partial t} & =\frac{1}{\rho c_{p}} \frac{\partial}{\partial z}\left(k(x(z, t)) \frac{\partial x(z, t)}{\partial z}\right) \\
& +\frac{b(z)}{\tau \int_{0}^{l} b(z) c(z) d z}\left[v(t)-y(t)-\frac{\tau}{\rho c_{p}} \int_{0}^{l} c(z) \frac{\partial}{\partial z}\left(k(x(z, t)) \frac{\partial x(z, t)}{\partial z}\right) d z\right]
\end{aligned}
$$

is internally stable.

\section{Application example}

A steel plate is heated (Fig. 1), before crossing a rolling mill, by thermal radiation applied only at the upper surface of the metallic plate [7] while the lower surface is assumed as adiabatic, i.e. no flux is applied, either for heating or cooling. The vertical sides of the plate are neglected so that finally the heat flux is applied only on the upper part of the perimeter of the cross-section. The heat flux $q^{\prime \prime}(t)$ is the manipulated variable and it is assumed to be distributed uniformly at the upper surface of the plate. At 
two positions, considered as $z=0$ and $z=l$, the plate is placed over rolls which are maintained at fixed temperatures and impose these conditions to the temperature of the plate itself. The control of the plate temperature is performed between these two cylinders.

To ensure a rolling of good quality, the heat flux $q^{\prime \prime}(t)$ is to be controlled in such a way that the temperature distribution, measured by a soft sensor that provides the spatial weighted average temperature $T_{m}(t)$, is kept at a specified temperature $T_{m}^{d}(t)$. The plate has a thickness $\delta$ of $10^{-2} \mathrm{~m}$. The distance $l$ is taken as $l=1 \mathrm{~m}$. The width of the plate is denoted as $r$.

The thermo-physical properties of the steel [28] are $\rho=7740 \mathrm{~kg} \cdot \mathrm{m}^{-3}$, $c=470 \mathrm{~J} \cdot \mathrm{kg}^{-1} \cdot \mathrm{K}^{-1}$, taken as constant, and the thermal conductivity $k(T)$ is a nonlinear function of temperature given as

$$
k(T)=23.9+4110^{-4} T+10^{-7} T^{2}
$$

where $T$ is given in Celsius.

First, to establish the model, consider the general case of a metal plate heated on all faces by a heat flux $q^{\prime \prime}$. It is assumed that the heat conduction is observed only along the $z$ axis, i.e. the model has only one dimension, 
and the temperature is uniform on a cross-section $A_{c}=r \delta$ of the plate. The energy balance formulated for a infinitesimal volume of the plate leads to the following PDE that describes the spatio-temporal evolution of plate temperature subject to the heat flux $q^{\prime \prime}(t)$

$$
A_{c} \rho c \frac{\partial T(z, t)}{\partial t}=A_{c} \frac{\partial}{\partial z}\left(k(T(z, t)) \frac{\partial T(z, t)}{\partial z}\right)+P q^{\prime \prime}(t)
$$

where $P$ is the heated perimeter corresponding to a cross-section and equal to: $P=2 r+2 \delta$.

In the present case, only the upper surface is heated by a radiative flux $q^{\prime \prime}$ between $z=0$ and $z=l$, the lower surface is adiabatic, the vertical faces are neglected, so that the heated perimeter is reduced to $P=r$ and the energy balance can be written as

$$
\frac{\partial T(z, t)}{\partial t}=\frac{1}{\rho c}\left[\frac{\partial}{\partial z}\left(k(T(z, t)) \frac{\partial T(z, t)}{\partial z}\right)+\frac{q^{\prime \prime}(t)}{\delta}\right]
$$

Both boundary conditions of the plate are assumed identical and of Dirichlet type

$$
T(0, t)=T(l, t)=T_{r} \quad \forall t \geq 0
$$


and the initial temperature distribution of the plate is assumed uniform

$$
T(z, 0)=T_{r} \quad \forall 0 \leq z \leq l
$$

with $T_{r}=800 \mathrm{~K}$.

The temperature distribution of the plate is assessed by the following measurement

$$
T_{m}(t)=\int_{0}^{l} c(z) T(z, t) d z
$$

with $c(z)=0.25 \cos (\pi / 2(1-z))$.

From the model (67), it follows that $b(z)=\delta^{-1}$, thus according to (38), the following control law results

$$
q^{\prime \prime}(t)=\frac{6 \delta \rho c}{\tau}\left[T_{m}^{d}(t)-T_{m}(t)-\frac{\tau}{\rho c} \int_{0}^{l} z(l-z) \frac{\partial}{\partial z}\left(k(T) \frac{\partial T(z, t)}{\partial z}\right) d z\right]
$$

For simulation purpose of the closed-loop system, the method of lines [38] is applied with evaluation of the spatial partial derivatives by means of finite differences based on $N=200$ discretization points. The integral terms in the control law are evaluated numerically using the trapezoidal method. The desired constant time $\tau$ is taken equal to $300 \mathrm{~s}$. The control is held constant 
over the sampling period equal to $30 \mathrm{~s}$. To avoid the consequences due to brutal set point steps, the set point $T_{m}^{d}(t)$ has been filtered by a first order filter with a time constant equal to $\tau_{f}=600 \mathrm{~s}$. Hence, in the control law (71) instead of set point $T_{m}^{d}(t)$, the filtered set point $T_{m_{f}}^{d}(t)$ defined by

$$
\tau_{f} \frac{d T_{m_{f}}^{d}(t)}{d t}+T_{m_{f}}^{d}(t)=T_{m}^{d}(t)
$$

is used.

To evaluate the performance of the control strategy, a set point step corresponding to $T_{m}^{d}(t)=160 \mathrm{~K}$ of the temperature is specified at $t=500 \mathrm{~s}$. Fig. 2 shows clearly that the controller behaves adequately and tracks perfectly the desired set point. Also, the control moves of the heat flux $q^{\prime \prime}(t)$ are smooth and physically acceptable (Fig. 3). An analogous remark could be made about the evolution of the temperature at different positions (Fig. 4). The two-dimensional and the three-dimensional plots of the temperature profiles given by Figs. 5 and 6, respectively, during the tracking of the imposed set point, confirm the good behavior of the controller. 


\section{Conclusion}

In this paper, a design approach of a distributed control, by input-output linearization, for a nonlinear diffusion system is proposed. The design approach is developed based on the Cole-Hopf transformation that converts the original nonlinear problem to a linear one, which allows to take advantage of some concepts from semi-group theory to prove the closed-loop stability. Thus, the distributed control law is derived using the concept of characteristic index from geometric control and it is shown that under certain assumptions concerning the control problem formulation, a first-order behavior results in closed loop between a desired reference and the controlled output.

The control performances of the proposed design approach are evaluated through numerical simulation by considering the problem of heating a steel plate in view of a rolling process. The obtained simulation results show the effectiveness of the developed control design.

This study demonstrates that the design of the control of PDE system, in the framework of geometric control, is a very successful control approach since it leads to a distributed control law that enhances the control performance by preserving the fundamental control properties, consequently the distributed nature of the PDE system. In addition, the use of the geometric control 
allows to take full advantage of the advances in control of linear lumped parameter systems to design robust controllers to the obtained linearized model. Nevertheless, the derived control law is infinite-dimensional, which requires to estimate the entire state of the system for on-line implementation. In practice, it is impossible to have the whole state, so its estimation from available measurements is necessary. The question of state estimation for nonlinear diffusion systems is an interesting challenge presently under the investigation of the authors.

\section{Appendix}

\subsection{Perturbations by bounded linear operators}

According to the following theorem [37, Theorem 1.1, page 76], the property of being a generator of a semi-group is preserved by the addition of bounded operators.

Theorem 1 Let $X$ be a Banach space and let $A$ be the infinitesimal generator of a $C_{0}$ semi-group $U(t)$ on $X$, satisfying $\|U(t)\|_{X} \leq M e^{\omega t}$. If $B$ is a bounded linear operator on $X$, then $A+B$ is the infinitesimal generator of a $C_{0}$ semigroup $V(t)$ on $X$, satisfying $\|V(t)\|_{X} \leq M e^{\left(\omega+M\|B\|_{X}\right) t}$. 


\subsection{Perturbation theorem}

The following perturbation theorem is a useful criterion to decide whether or not a given family of infinitesimal generators is stable [37, Theorem 1.1, page 76$]$.

Theorem 2 Let $A$ be a stable family of infinitesimal generators with stability constants $M$ and $\omega$. Let $B$ be bounded linear operators on $X$. If $\|B(t)\|<$ $K$ then $A+B$ is a stable family of infinitesimal generators with stability constants $M$ and $\omega+M K$.

\subsection{Bounded operator}

Definition 1 A linear operator $A$ from $X$ to $Y$ is said to be bounded if there exists a constant $C$ such that

$$
\|A x\|_{Y} \leq C\|x\|_{X}
$$

If no such $C$ exists, the operator is said to be unbounded. 


\section{References}

[1] Christofides PD. Control of nonlinear distributed process systems: Recent developments and challenges. AIChE Journal 2001; 47(3):514-518.

[2] Padhi R, Faruque Ali S. An account of chronological developments in control of distributed parameter systems. Annual Reviews in Control 2009; 33(1):59-68.

[3] Curtain RF, Zwart HJ. An Introduction to Infinite-Dimensional Linear Systems Theory. Springer-Verlag: New York, 1995.

[4] Bensoussan A, Da Prato G, Delfour MC, Mitter SK. Representation and Control of Infinite Dimensional Systems. Second edn., Birkhäuser: Boston, 2007.

[5] Chen G, Lascieka I, Zhou J ( (eds.)). Control of Nonlinear Distributed Parameter Systems. Marcel Dekker: New York, 2001.

[6] Christofides PD. Nonlinear and robust control of PDE systems: methods and applications to transport-reaction processes. Birkhäuser: Boston, 2001. 
[7] Ray WH. Advanced Process Control. Butterworths Publishers: Boston, 1989.

[8] Baker J, Christofides PD. Finite-dimensional approximation and control of nonlinear parabolic PDE systems. International Journal of Control 2000; 73(5):439-456.

[9] Christofides PD, Daoutidis P. Finite-dimensional control of parabolic PDE systems using approximate inertial manifolds. Journal of Mathematical Analysis and Applications 1997; 216(2):398-420.

[10] Liu W. Elementary feedback stabilization of the linear reactionconvection-diffusion equation and the wave equation. Springer-Verlag: Berlin, 2010.

[11] Li HX, Qi C. Modeling of distributed parameter systems for applications-A synthesized review from time-space separation. Journal of Process Control 2010; 20(8):891-901.

[12] Dubljevic S, Christofides PD, Kevrekidis IG. Distributed nonlinear control of diffusion-reaction processes. International Journal of Robust and Nonlinear Control 2004; 14(2):133-156. 
[13] Demetriou MA, Kazantzis N. Compensation of spatiotemporally varying disturbances in nonlinear transport processes via actuator scheduling. International Journal of Robust and Nonlinear Control 2004; 14(2):181197.

[14] Demetriou MA, Kazantzis N. A new integrated output feedback controller synthesis and collocated actuator/sensor scheduling framework for distributed parameter processes. Computers \& Chemical Engineering 2005; 29(4):867-876.

[15] Lou Y, Christofides PD. Feedback control of surface roughness in sputtering processes using the stochastic Kuramoto-Sivashinsky equation. Computers \& Chemical Engineering 2005; 29(4):741-759.

[16] Godasi S, Karakas A, Palazoglu A. Control of nonlinear distributed parameter processes using symmetry groups and invariance conditions. Computers \& Chemical Engineering 2002; 26(7-8):1023-1036.

[17] Christofides PD, Daoutidis P. Feedback control of hyperbolic PDE systems. AIChE Journal 1996; 42(11):3063-3086. 
[18] Wu W, Liou CT. Output regulation of nonisothermal plug-flow reactors with inlet perturbations. Computers \& Chemical Engineering 2001; 25(2-3):433-443.

[19] Shang H, Forbes JF, Guay M. Feedback control of hyperbolic distributed parameter systems. Chemical Engineering Science 2005; 60(4):969-980.

[20] Maidi A, Diaf M, Corriou JP. Boundary geometric control of a countercurrent heat exchanger. Journal of Process Control 2009; 19(2):297-313.

[21] Maidi A, Diaf M, Corriou JP. Boundary geometric control of a heat equation. European Control Conference (ECC'09) 2009; August 2326, 2009, Budapest, Hungary:4677-4682.

[22] Maidi A, Diaf M, Corriou JP. Boundary control of a parallel-flow heat exchanger by input-output linearization. Journal of Process Control 2010; 20(10):1161-1174.

[23] Maidi A, Corriou JP. Distributed feedback design for systems governed by the wave equation. International Journal of Control 2011; 84(8):1417-1429. 
[24] Maidi A, Corriou JP. Boundary control of nonlinear distributed parameter systems by input-output linearization. 18th IFAC World Congress 2011; August 28-September 02, 2011, Milan, Italy:10 910-10 915.

[25] Renardy M, Rogers RC. An introduction to partial differential equations, Texts in Applied Mathematics, vol. 13. Second edn., Springer-Verlag: New York, 2004.

[26] Atkinson K, Han W. Theoretical numerical Analysis. A functional analysis framework, Texts in Applied Mathematics Series, vol. 39. Third edn., Springer-Verlag, 2009.

[27] Vadasz P. Analytical solution to nonlinear thermal diffusion: Kirchhoff versus Cole-Hopf transformations. Journal of Heat Transfer 2010; 132(12):121 302.1-121306.6.

[28] Taler J, Duda P. Solving Direct and Inverse Heat Conduction Problems. Springer-Verlag: Berlin, 2006.

[29] Arpaci VS. Conduction Heat Transfer. Mechanics and Thermodynamics Series, Addison-Wesley: London, 1966. 
[30] Carslaw HS, Jaeger JC. Conduction of Heat in Solids. Second edn., Oxford University Press: Oxford, UK, 1959.

[31] Isidori A. Nonlinear control systems. Third edn., Springer-Verlag: New York, 1995.

[32] Corriou JP. Process control - Theory and applications. Springer-Verlag: London, 2004.

[33] Balas MJ. Finite-dimensional control of distributed parameter systems by Galerkin approximation of infinite dimensional controllers. Journal of Mathematical Analysis and Applications 1986; 114(1):17-36.

[34] Kravaris C, Kantor JC. Geometric methods for nonlinear process control. 1. Background. Industrial \& Engineering Chemistry Research 1990; 29(12):2295-2310.

[35] Kravaris C, Kantor JC. Geometric methods for nonlinear process control. 2. Controller synthesis. Industrial \& Engineering Chemistry Research 1990; 29(12):2310-2323.

[36] El Jai A, Amouroux M. Automatique des Systèmes Distribués. Hermès: Paris, 1990. 
[37] Pazy A. Semigroups of Linear Operators and Applications to Partial Differential Equations. Springer-Verlag: New York, 1983.

[38] Vande Wouwer A, Saucez P, Schiesser WE. Adaptive method of lines. Chapman and Hall/CRC: New York, 2001. 


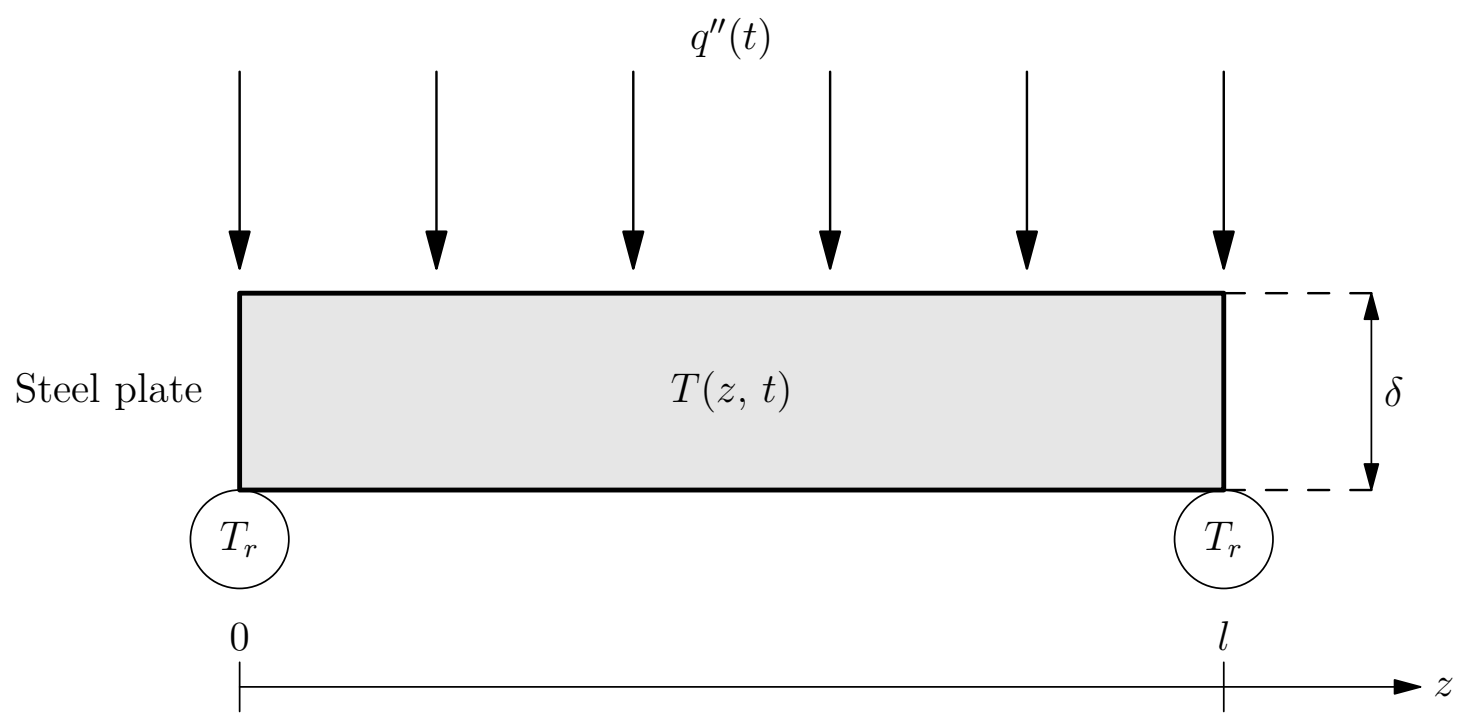

Figure 1: Heating of steel plate (sectional representation).

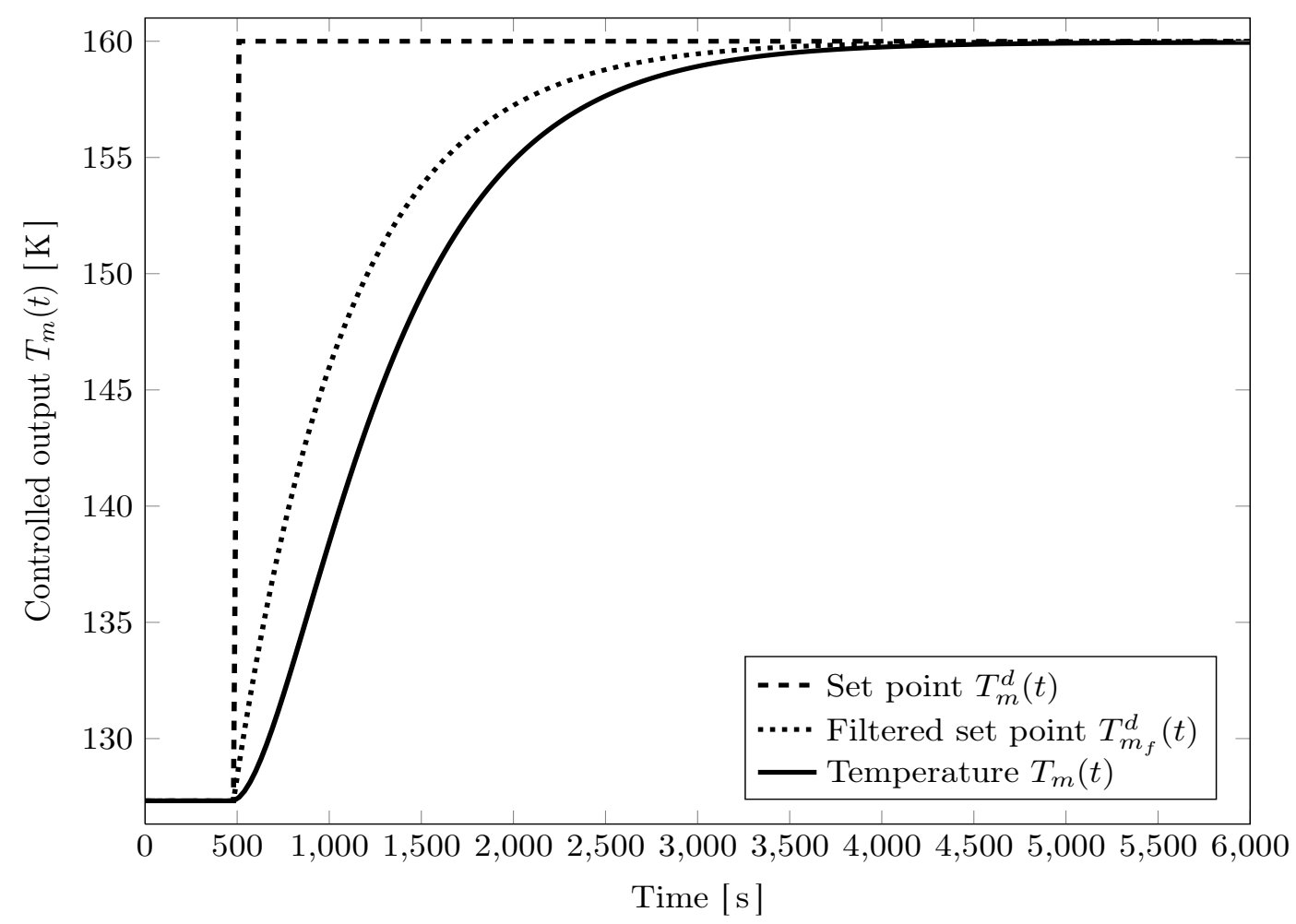

Figure 2: Evolution of the controlled temperature $T_{m}(t)$. 


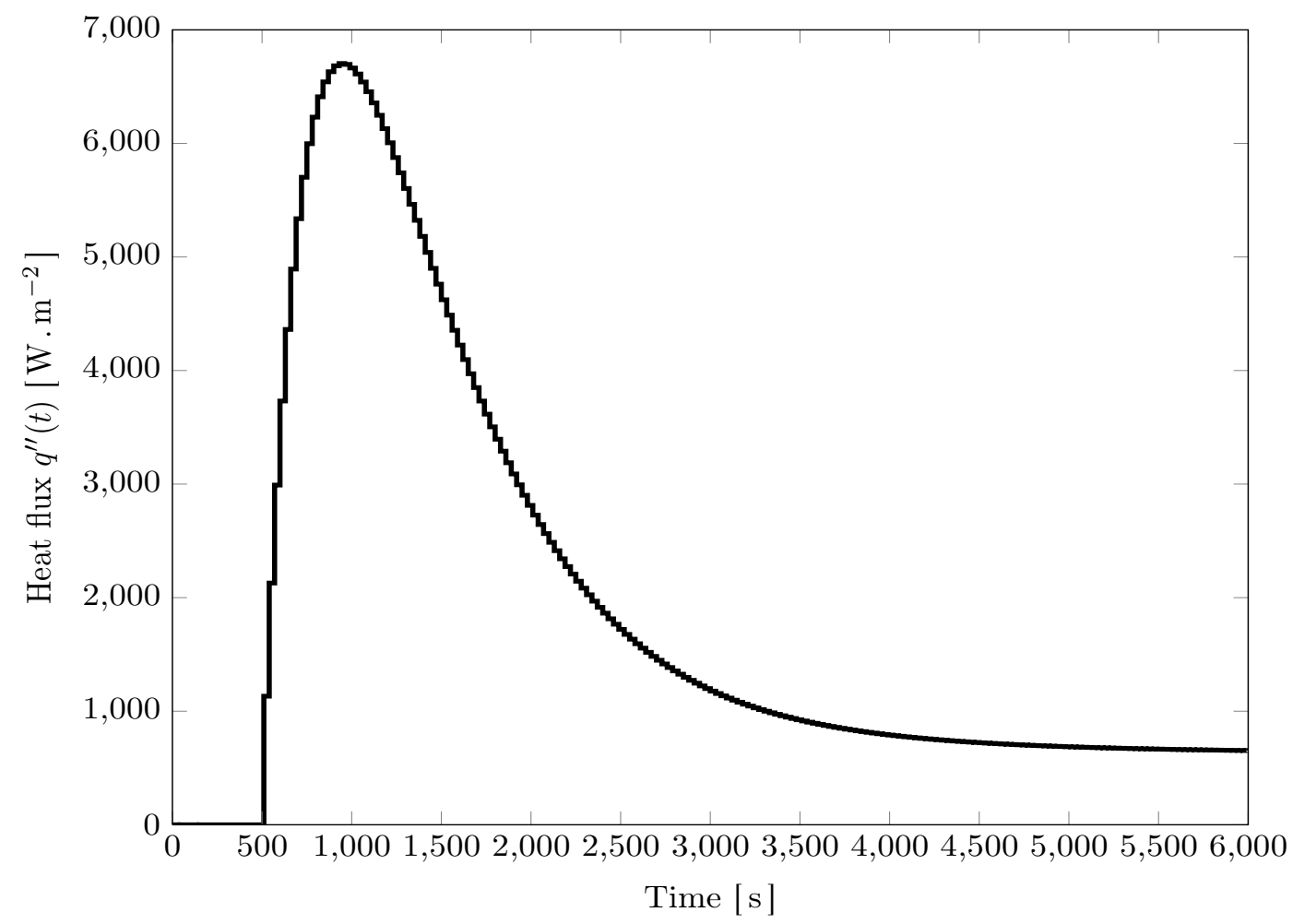

Figure 3: Evolution of the heat flux $q^{\prime \prime}(t)$. 


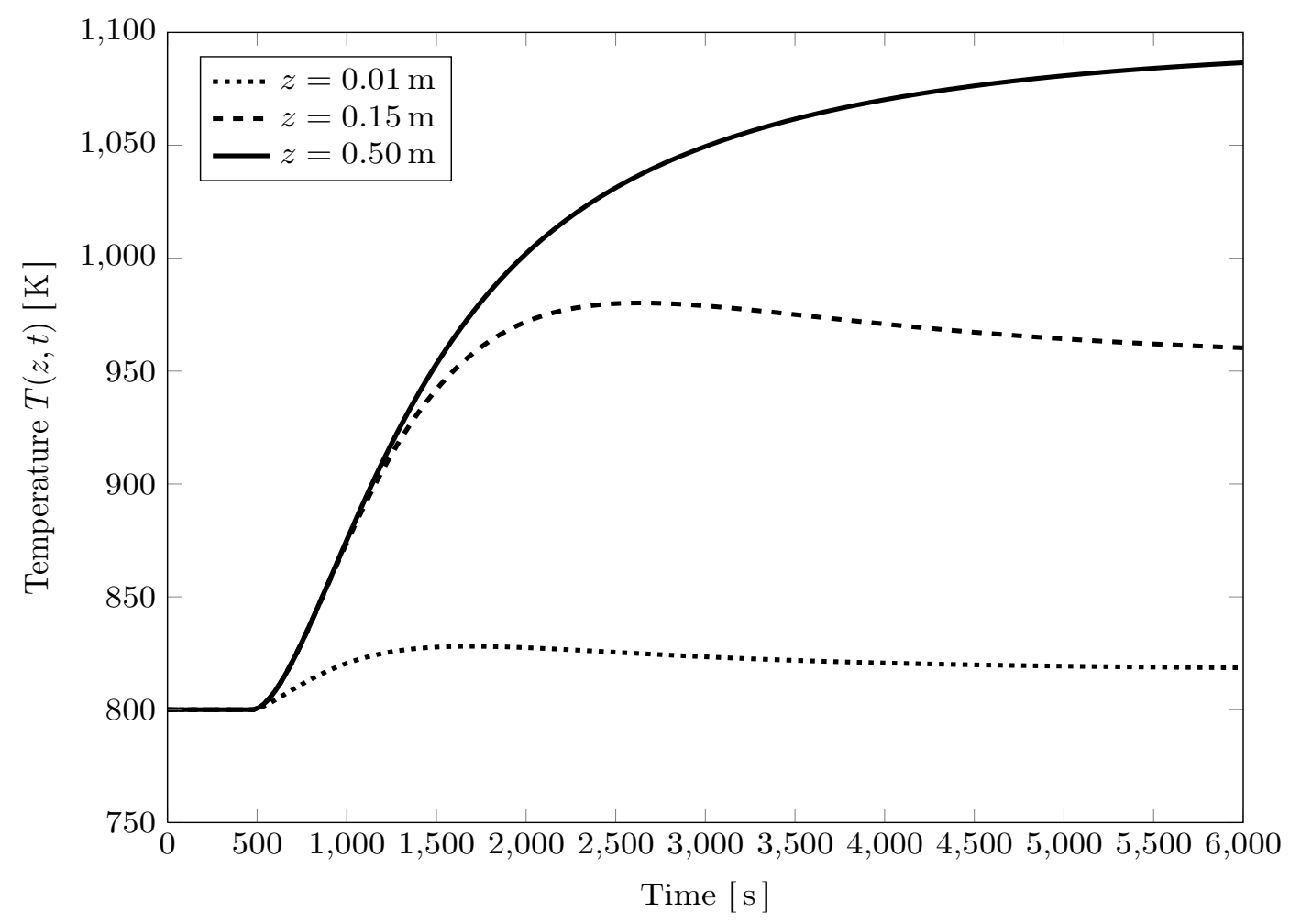

Figure 4: Variation of temperature at different locations along the plate. 


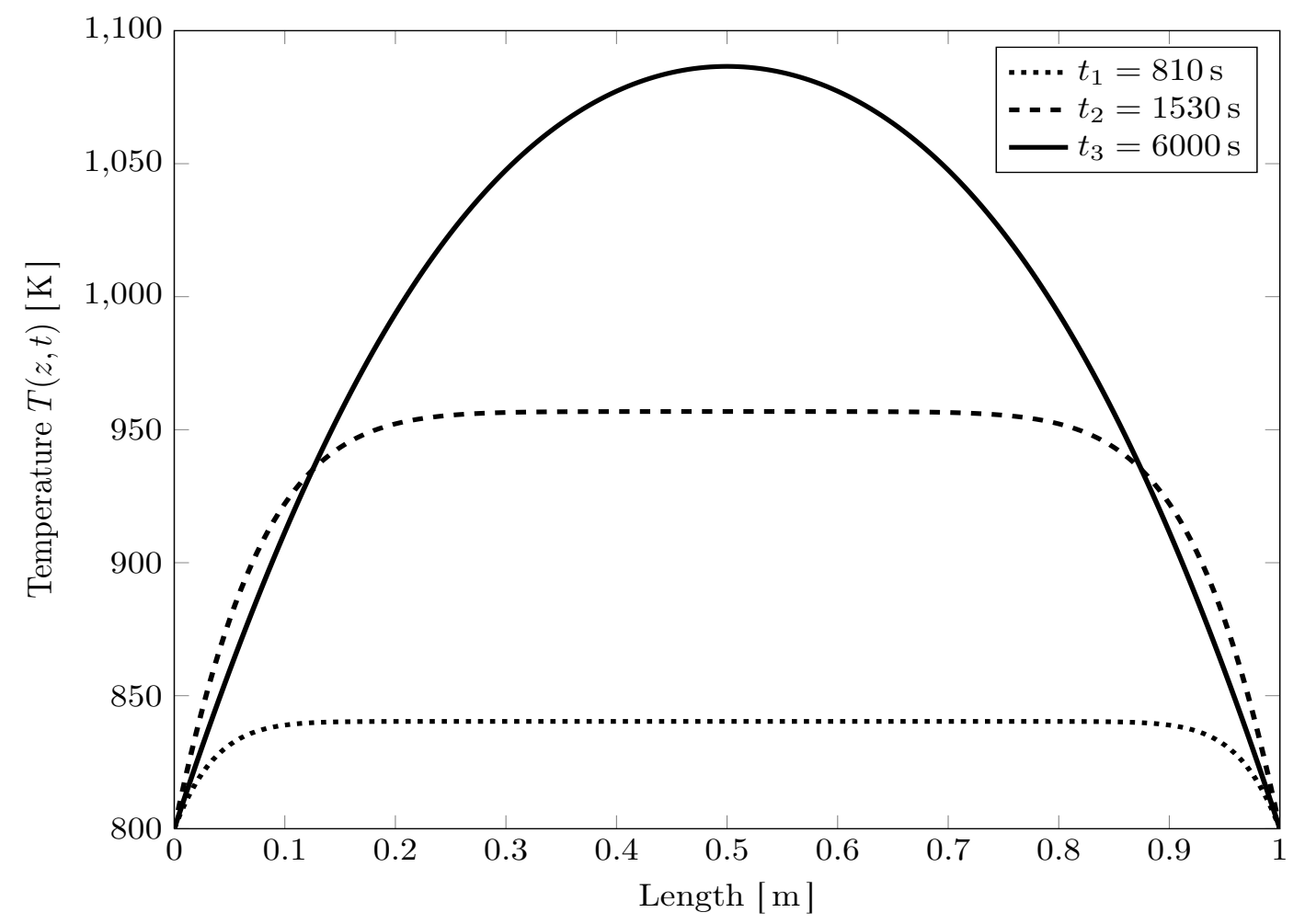

Figure 5: Temperature profiles at different time instants. 


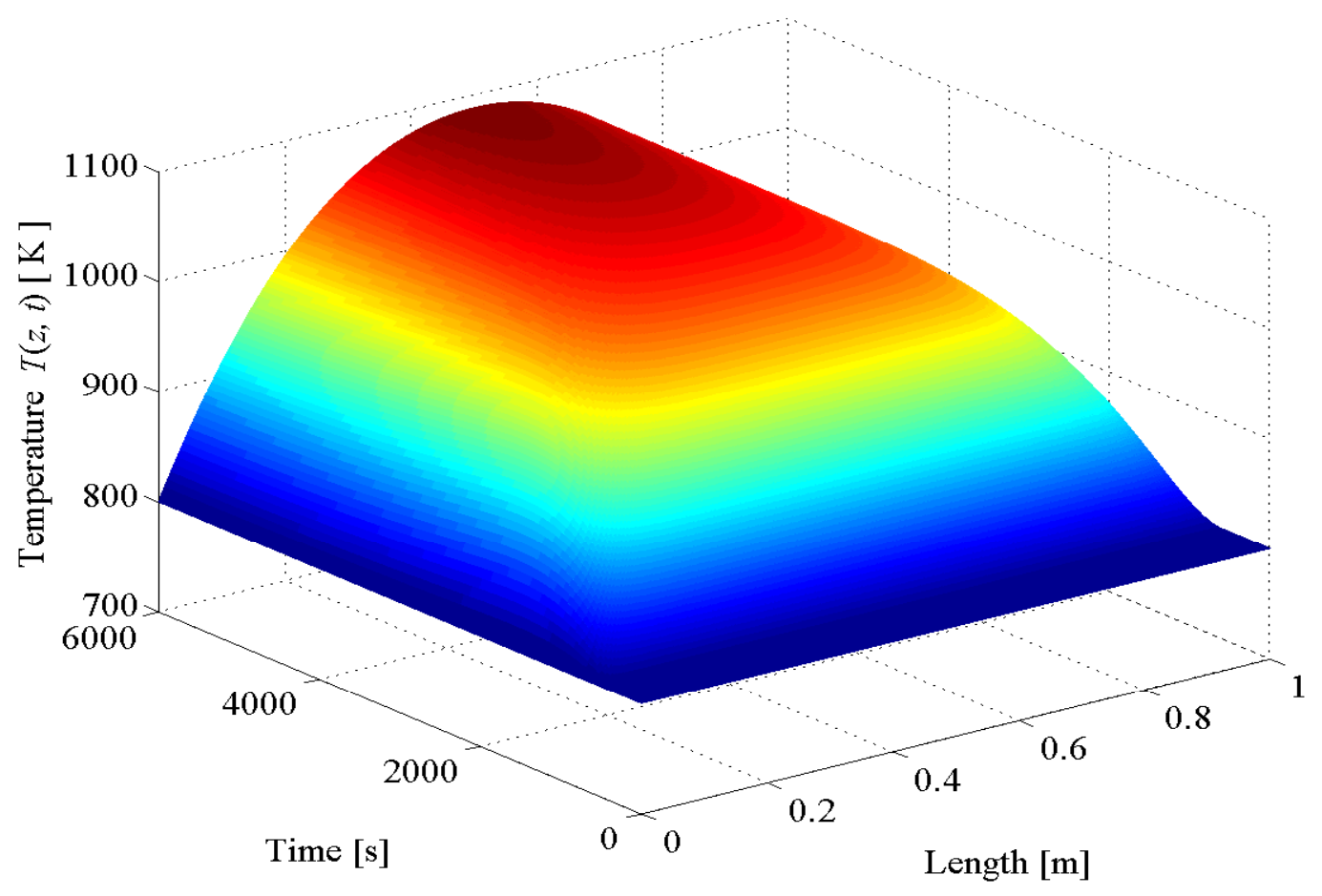

Figure 6: Temperature spatial profile. 